\title{
Use of Quantitative Ultrasound Scans of the Calcaneus to Diagnose Osteoporosis in Patients with Rheumatoid Arthritis
}

\author{
Jacqueline R. Cryer, MSc* \\ Simon J. Otter, MSc† \\ Catherine J. Bowen, MSc‡
}

\begin{abstract}
Background: Patients with rheumatoid arthritis are recognized as being at risk for osteoporosis as a result of the disease process as well as the medication used to treat it. This study was conducted to consider the use of calcaneal scanning with quantitative ultrasound-contact ultrasound bone analysis (CUBA)-to diagnose osteoporosis in patients with rheumatoid arthritis.

Methods: Forty-six patients (11 men and 35 women) with established rheumatoid arthritis underwent dual-energy $x$-ray absorptiometry (DEXA) of the nondominant wrist and CUBA of the nondominant heel. Sensitivity, specificity, and positive and negative predictive values were used to determine the correlation between osteoporosis as diagnosed by the CUBA heel scan compared with the DEXA wrist scan given that DEXA is widely seen as the gold standard for the diagnosis of osteoporosis.

Results: The CUBA heel scan revealed a sensitivity of $90 \%$ and a specificity of $44 \%$ for a diagnosis of osteoporosis compared with DEXA. The positive predictive value of the CUBA scan was $31 \%$, and the negative predictive value was $94 \%$. Therefore, if normal bone density is found using CUBA, there is $94 \%$ certainty this is correct. However, if osteoporosis is diagnosed using CUBA, there is only $31 \%$ certainty this is correct. In such instances a secondary scan using a different method (eg, DEXA) would be required. Future work should consider the effect of minor alterations to the equipment or scanning protocol, because this may improve diagnosis.

Conclusions: The CUBA unit could be used as a primary screening device. Given the cost and accessibility issues associated with DEXA, quantitative ultrasound may have a role in screening for osteoporosis in the primary-care setting to determine the most appropriate routes of referral for patients requiring further investigations. (J Am Podiatr Med Assoc 97(2): 108-114, 2007)
\end{abstract}

Osteoporosis is a growing problem worldwide. In 1997 a World Health Organization report ${ }^{1}$ estimated that approximately 380 million people 65 years or older were affected by osteoporosis, and this figure is expected to rise to 690 million by 2020 . Considering that osteoporosis affects mostly elderly people (including approximately 1 in 3 postmenopausal women), ${ }^{2}$ it

*Department of Podiatry, Eastbourne Downs Primary Care Trust, Eastbourne, England.

$\uparrow$ School of Health Professions, University of Brighton, Leaf Hospital, Eastbourne, England.

$\$$ School of Health Professions and Rehabilitation Science, University of Southampton, Highfield, England.

Corresponding author: Jacqueline R. Cryer, MSc, Department of Podiatry, Eastbourne Downs Primary Care Trust, Avenue House, The Avenue, Eastbourne BN21 3XY, England. is not surprising that the World Health Organization Bone and Joint Decade predicts that the number of hip fractures associated with osteoporotic changes will increase from 1.7 million in 1990 to 6.3 million by 2050 unless preventive programs are introduced. ${ }^{3}$ In the United Kingdom, as elsewhere, the morbidity associated with osteoporosis not only diminishes quality of life ${ }^{4,5}$ but also incurs significant financial costs to health-care providers. For example, in 1998 Dolan and Torgerson ${ }^{6}$ reported that osteoporotic fractures cost the National Health Service approximately $\$ 942$ million annually. More recently, the National Service Framework for Older People ${ }^{7}$ indicated that 14,000 people died as a direct result of an osteoporotic hip 
fracture and that $50 \%$ of those who survived cannot live independently. These statistics support the view proposed by the 1997 World Health Organization report $^{1}$ that "pain, suffering, loss of physical capacity and social impairment" were the prices paid by those living longer and consequently at increased risk for chronic musculoskeletal complaints, including osteoporosis and rheumatoid arthritis.

Osteoporosis is not only a disease of the frail elderly. It has a multifactorial etiology, reviewed in detail elsewhere. ${ }^{8-10}$ A significant proportion of patients with rheumatoid arthritis are subsequently diagnosed as having osteoporosis, the etiologic factors of which are reviewed in Table 1. ${ }^{11-15}$ The pathogenesis of rheumatoid arthritis-associated osteoporosis is a complex, multifactorial process that includes the effect of proinflammatory cytokines, medication, and postmenopausal hormone loss, making risk assessment difficult. ${ }^{16}$ People with rheumatoid arthritis have a greater risk of fracture associated with reduced bone mineral density, particularly in connection with corticosteroid treatment. ${ }^{17-20}$ However, the precise hierarchy of risk factors for osteoporotic fractures requires further epidemiologic research. ${ }^{21,22}$

The prevention and management of osteoporosis is a key aim of the National Service Framework for Older People ${ }^{7}$ because it can have a significant effect on the number and cost of fractures. Beardsworth and Purdie ${ }^{23}$ consider that the immediate future lies in selectively screening individuals with recognized risk factors. In primary care, preventing the first fracture by identifying and treating those at risk for osteoporosis is also a key aim. ${ }^{24} \mathrm{~A}$ variety of treatment strategies are reported in the literature, including dietary modification, calcium and vitamin D supplementation, weightbearing exercise, hormone therapy, and bisphosphonate use. ${ }^{16,25}$ In terms of identification, osteoporosis is defined on the basis of bone density. The quantitative definition of osteoporosis is a bone mineral density value less than 2.5 SD below the adult mean value. ${ }^{26}$ The current gold standard for

Table 1. Etiologic Factors Associated with Osteoporosis in Rheumatoid Arthritis

Immobility and habitual underuse of full range of motion

Periarticular osteoporosis reflecting disease activity

Loss of systemic bone remodeling

Secondary to long-term corticosteroid use

Nutritional deficiency

Methotrexate use

Source: Data from Refs. 11-15. quantifying bone mineral density is dual-energy x-ray absorptiometry (DEXA), which provides high resolution and is quick, precise, and accurate. ${ }^{11}$ However, DEXA is rarely used in the primary-care setting, where most regular patient contacts occur for individuals at high risk for osteoporosis, partly because of the need for bulky and expensive equipment. More recently, newer screening tools have become available, in particular noninvasive quantitative ultrasound machines, which are cheaper, smaller, and more portable, making them ideal for use in primary-care settings. Njeh et $\mathrm{al}^{27}$ suggest that because quantitative ultrasound is an accepted tool for the measurement of bone mineral density, it could be used to screen for bone changes in patients with rheumatoid arthritis. However, others argue that further research needs to be undertaken regarding the diagnostic precision of quantitative ultrasound before it is used as a screening tool in widespread clinical practice. ${ }^{11}$ The aim of this study was to determine whether contact ultrasound bone analysis (CUBA) calcaneal scanning is a reliable measure for osteoporosis in people with rheumatoid arthritis.

\section{Methods}

\section{Patients and Setting}

Fifty patients with a diagnosis of rheumatoid arthritis (according to the 1987 American Rheumatism Association criteria ${ }^{28}$ ) were recruited from a rheumatology outpatient department; sampling was purposeful and convenience based. Patients with concomitant systemic disease, including previous cerebrovascular accident, diabetes mellitus, and inflammatory arthropathies other than rheumatoid arthritis, were excluded, as were amputees, those with previous fractures of the wrist or calcaneus, and pregnant women. Patients underwent a DEXA scan of the nondominant wrist and a CUBA scan of the heel (on the same side as the wrist scan). Data were collected in the hospital outpatient department for 2 months, coinciding (where possible) with patients' normal appointments.

All of the participants gave written informed consent, and ethical approval was granted by Brighton \& Hove Primary Care Group research ethics committee for this study. Bone density measurements were performed as part of ongoing local research into the role of primary-care providers undertaking routine screening of "at-risk" populations to facilitate more appropriate referral and care pathways. This study used a comparative trial design focusing on within-group comparisons.

Celebrating 100 years of continuous publication:1907-2007 


\section{Clinical Data}

Participants provided demographic details and completed an osteoporosis status questionnaire of up to 21 items, including open and closed questions that considered the symptoms of osteoporosis, family history, current treatment for osteoporosis, and previous bone density measurements.

\section{Bone Density Measurements}

Bone mineral density and bone mineral content were measured at the distal forearm using a peripheral densitometer (model DTX-200; Churchill Medical Ltd, Northants, England). The forearm is placed in a shaped container where a high-voltage $(55-\mathrm{kV})$ pencil-beam $\mathrm{x}$ ray source is located on one side of the forearm and a detector on the other side. In rheumatoid arthritis, the hand and forearm is a principal site of inflammation and synovitis. Bone mineral density measurements of this site are considered important because levels of periarticular osteoporosis have been shown to reflect disease activity. ${ }^{29}, 30$ The participant's nondominant side was measured because differences in bone density between dominant and nondominant hands may yield higher values on the dominant side. ${ }^{31,32}$

Quantitative ultrasound was used to measure bone ultrasonic attenuation at the calcaneus using the CUBA clinical instrument (McCue Ultrasonic, Winchester, England). Two transducers (receiving and emitting) faced with silicone rubber coupling pads were placed in direct contact on either side of the calcaneus using a coupling gel (Fig. 1). Participants underwent single scan measurements of the calcaneus on the same side

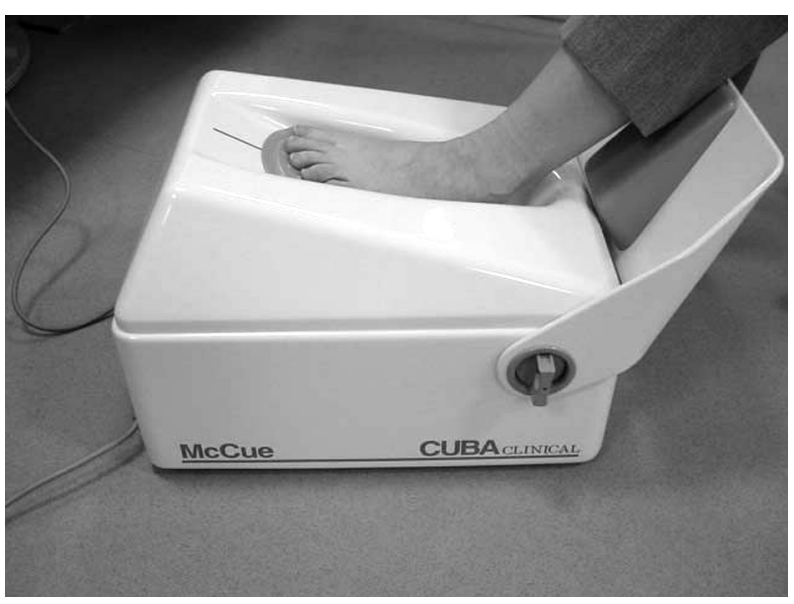

Figure 1. Contact ultrasound bone analysis (CUBA) scanner. as the DEXA wrist scan. Characterization parameters measure the velocity of sound and broadband ultrasonic attenuation. Owing to its larger surface area, trabecular bone is metabolically more active and so more readily affected by factors that may cause bone loss. ${ }^{33}$ Therefore, bone loss is most rapid in areas of the skeleton with the highest proportion of trabecular bone. ${ }^{34}$ In common with other sites usually assessed for osteoporotic changes, such as the vertebrae, the calcaneum is $95 \%$ trabecular bone, making it a suitable site for determining osteoporotic changes.

\section{Repeatability and Reliability}

Before patient assessment using the DEXA unit, a quality-control phantom scan was performed on a phantom with a known bone mineral content of $3.618 \mathrm{~g}$ to ensure correct calibration. In accordance with manufacturer guidelines, a standard phantom was used to calibrate the CUBA unit before the study. Intratester and intertester reliability tests did not demonstrate significance at the 5\% level, suggesting that the primary researcher (J.R.C.) was producing consistent measurements on the same patients using the same machine.

\section{Statistics}

The results of the DEXA unit and the bone ultrasonic attenuation $t$ score of the CUBA unit were compared using the Spearman rank correlation coefficient to determine the relationship between the values of these two variables. To assess the usefulness of the CUBA unit in clinical practice, the sensitivity, specificity, and positive and negative predictive values were also calculated. Sensitivity refers to the proportion of participants with osteoporosis as determined by a DEXA scan and then correctly identified by the CUBA scan, whereas specificity is the proportion of participants without osteoporosis correctly identified by the CUBA scan. These data are presented as percentages. The positive predictive value refers to the proportion of participants with positive CUBA scan findings who have osteoporosis. The negative predictive value is the proportion of participants with negative CUBA scan findings who do not have osteoporosis; both data sets are expressed as percentages.

\section{Results}

Forty-six participants completed the trial and four withdrew (one moved away, one had an emergency surgical admission, and two did not complete data collection). The demographic and clinical character- 
istics of the participants are listed in Table 2. The correlations obtained for the DEXA and CUBA scanners suggest a weak positive association between the two variables $(\rho=0.341 ; P=.02)$ (Fig. 2 ). The DEXA wrist scans identified $78 \%$ of the participants as having normal bone density and the remaining $22 \%$ as having osteoporosis. In contrast, the CUBA heel scans identified $37 \%$ of the patients as having normal bone density and the remaining $63 \%$ as having osteoporosis (Table 3). Currently, DEXA is the accepted method of diagnosing osteoporosis; therefore, these data suggest that of the CUBA scanner findings, $20 \%$ are true-positive osteoporosis results and 35\% are true-negative results, with $43 \%$ being false-positive osteoporosis results and $2 \%$ being false-negative results. Using the clinical data from Table 2, sensitivity of the CUBA unit was high (90\%) but specificity was lower (44\%); the positive predictive value of the CUBA unit was $31 \%$ and the negative predictive value was $94 \%$.

\section{Discussion}

The results of this study suggest that the CUBA unit may have limited usefulness in diagnosing osteoporosis from a calcaneal scan in a population with rheumatoid arthritis. Specificity for diagnosing bone mineral density-defined osteoporosis was low (31\%), but sensitivity remained high (94\%). Therefore, these results indicate that if a normal bone density value is obtained using the CUBA unit, there is 94\% certainty this is correct. However, if osteoporosis is diagnosed, there is only $31 \%$ certainty this is correct, given a re-

Table 2. Demographic and Clinical Characteristics of the 46 Study Participants

\begin{tabular}{lc}
\hline Characteristic & Value \\
\hline Sex (No. [\%]) & $11(24)$ \\
M & $35(76)$ \\
F & $61(26-91)$ \\
Age (mean [range]) (years) & $166.9(147.3-188)$ \\
Height (mean [range]) (cm) & $72.3(43.5-111)$ \\
Body weight (mean [range]) $(\mathrm{kg})$ & 35 \\
Current or previous smokers (\%) & 21 \\
Previous fracture (other than wrist & \\
$\quad$ or calcaneum) $(\%)_{\text {Previous corticosteroid use (\%) }}^{a}$ & 42 \\
Previous bisphosphonate therapy (\%) & 54 \\
${\text { Right-handed }(\%)^{b}}^{b}$ & 83 \\
\hline
\end{tabular}

${ }^{a}$ No participants were currently taking corticosteroids or bisphosphonates.

${ }^{b}$ All of the scans were undertaken on the nondominant side.

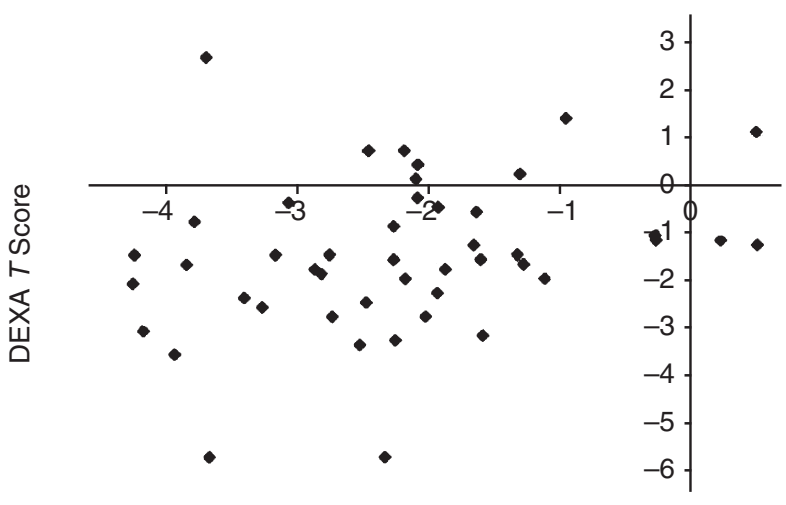

CUBA (BUA) T Score

Figure 2. $T$ scores obtained using the dual-energy $x$ ray absorptiometry (DEXA) and contact ultrasound bone analysis (CUBA) scanners. BUA indicates bone ultrasonic attenuation.

ported precision error of $\pm 1 \%$ for the DEXA unit. 35 The results of this study indicate that there could be a useful role for the CUBA unit not as a screening tool for osteoporosis but as a screening tool for normal bone density. Primary-care clinicians may increasingly incorporate ultrasound densitometry into their practice because of its low cost and portability, giving a wider applicability (eg, use in people's homes), something DEXA units cannot currently offer. ${ }^{36,37}$ In addition, some patients with advanced disease may have severe ulnar deviation, making it impossible to use portable DEXA wrist scanners, or may be unsuitable for DEXA scanning owing to the presence of a prosthetic joint used in total hip replacement. In such cases, using the CUBA unit to screen for normal bone density may be more appropriate.

The correlation between the CUBA and DEXA units was weakly positive $(\rho=0.341)$. This correlation is broadly similar to that of other studies in which different anatomical sites were assessed. Imamoto et $\mathrm{al}^{38}$

$\begin{aligned} & \text { Table 3. Relationship Between Results of the DEXA Wrist } \\
& \text { Scan and the CUBA Heel Scan in the 46 Study Participants }\end{aligned}$
\begin{tabular}{lccc}
\multicolumn{3}{c}{ DEXA } \\
\cline { 2 - 3 } & Osteoporosis & Normal & Total \\
\hline CUBA & & 20 & 29 \\
Test positive & 9 & 16 & 17 \\
Test negative & 1 & 36 & 46 \\
Total & 10 & 36
\end{tabular}

Abbreviations: DEXA, dual-energy x-ray absorptiometry; CUBA, contact ultrasound bone analysis.

Celebrating 100 years of continuous publication:1907-2007 
reported in cadaveric studies a correlation between bone mineral density and bone ultrasonic attenuation of $r=0.39$. Tromp et al $^{39}$ compared quantitative ultrasound measurements of the calcaneum with DEXA measurements of the lumbar spine, femoral neck, greater trochanter, and total body, yielding $r$ values of $0.41,0.35,0.45$, and 0.47 , respectively. Regarding sensitivity and specificity, Naganathan et $\mathrm{al}^{40}$ reported a sensitivity of CUBA calcaneal scanning of $9 \%$ to $47 \%$ and a specificity of $88 \%$ to $100 \%$. However, these studies compared quantitative ultrasound of the calcaneum with axial DEXA measurements. It is recommended that appendicular and axial skeletal sites are measured (most commonly the lumbar spine and hip ${ }^{10}$ ); this approach is expensive, requires bulky equipment, and necessitates using ionizing radiation. ${ }^{41}$

In this study, a higher proportion of participants were identified as having osteoporosis using the CUBA scanner (63\%) compared with DEXA (22\%). Radiographic studies ${ }^{42-45}$ have repeatedly shown that in rheumatoid arthritis, periarticular erosions seem to occur earlier and possibly more frequently in the feet than in the hands. Considering the interaction between inflammatory synovitis and mechanical stress, ${ }^{46}$ it is possible that participants in this study have more osteoporotic damage in their feet. This has led to further speculation ${ }^{45}$ that periarticular erosions in the foot may be more common owing to the subluxation of joints commonly observed in the feet of individuals with rheumatoid arthritis.

Involvement of the rearfoot in rheumatoid arthritis has been reported to affect $42 \%$ to $80 \%$ of patients with rheumatoid arthritis, ${ }^{47-49}$ often leading to a valgus deformity of the rearfoot. Although not measured in this study, valgus calcaneal drift may have implications for quantitative ultrasound studies of the calcaneum. Gluer et $\mathrm{al}^{50}$ found that the bone ultrasonic attenuation of the calcaneum could be up to $50 \%$ larger depending on the direction from which quantitative ultrasound was measured. Cadaveric studies ${ }^{51}$ have found different bone mineral densities between the medial and lateral sides of the calcaneus using quantitative ultrasound. These findings may be partially explained by the gait alterations seen in patients with rheumatoid arthritis, where significant foot involvement is reported to be an important cause of walking difficulty. ${ }^{47,} 52$ Joint range of motion and immobilization due to either recurrent flares of the disease or deformation of joints could lead to loss of mobility, thus reducing the loading response required to maintain healthy bone. Bone trabeculae thin with age, and bone that is no longer subjected to biomechanical stress will undergo rapid resorption. ${ }^{53}$ The present study investigated the correlation between two pe- ripheral scanning methods because it is possible that axial skeletal sites are not the most relevant sites to scan for osteoporosis in people with rheumatoid arthritis given its pathogenesis. ${ }^{27}$

In terms of the methodologic quality of this study, there are three areas worthy of discussion. Should the study be repeated, the inclusion of an axial DEXA measurement (eg, lumbar spine or hip) would be valuable. This may help determine the relative importance of issues such as the positioning of fixed transducers and the effect of calcaneal or ulnar deviation on the results. This is important because DEXA measures in two dimensions, ${ }^{10}$ whereas quantitative ultrasound is thought to measure in three dimensions. ${ }^{41}$ The addition of a linear potentiometer to measure the aperture between transducers would potentially allow for bone width to be calculated, thus bringing a threedimensional element to bone density measurement.

The use of an instrument to measure function (eg, the Health Assessment Questionnaire ${ }^{54}$ or the Foot Function Index ${ }^{55}$ ) may have 1) provided information as to the amount of weightbearing activity undertaken by the participant and, therefore, the risk of osteoporosis and 2) identified any participants currently experiencing a flare of the disease because confined areas of inflammation can induce localized osteopenia, thus influencing bone density measurements. ${ }^{29}$

Finally, the management of rheumatoid arthritis itself may also have a bearing on the results of this study. In the contemporary management of rheumatoid arthritis, glucocorticosteroid medication may be used to reduce disease activity in the short term, or in lower doses to control mild disease. However, the wide range of adverse effects (including osteoporosis) and the introduction of newer disease-modifying agents now make corticosteroids unsuitable for longterm use. ${ }^{11,56}$ The osteoporotic adverse effects of corticosteroids are most apparent in the regions of the skeleton with high trabecular bone content.57 Therefore, there could be a greater degree of bone loss in the calcaneum (95\% trabecular bone) compared with the wrist, which contains $20 \%$ trabecular bone in the radius. More precise information relating to the dose and duration of glucocorticosteroid drugs might be worthy of subgroup analysis. However, ascertaining details from participants that were precise enough to facilitate further analysis proved to be difficult.

Recommendations for the management of osteoporosis involve early diagnosis and appropriate treatment. ${ }^{58}$ Identifying people who have osteoporosis before a fracture occurs and enabling targeted preventive therapy remains a challenge, particularly in rheumatoid arthritis, where there is a need for a cost-effective reproducible marker of osteoporotic progression. ${ }^{27}$ 


\section{Conclusion}

Although the CUBA scans in this study may not be suitable for diagnosing osteoporosis, they may have a role in identifying individuals with normal bone density. To improve the diagnostic potential of CUBA, further work is recommended regarding calcaneal and transducer positioning of CUBA units. In addition, consideration should be given to the possibility of a wider role for quantitative ultrasound scanning for normal bone density in people with rheumatoid arthritis.

Acknowledgments: Ian Lloyd for technical assistance with the CUBA scanner and Kevin Lucas, DPhil, and Farina Hashmi, PhD, for their helpful comments on the manuscript.

Financial Disclosures: None reported.

Conflict of Interest: None reported.

\section{References}

1. World Health Organization: The World Health Report 1997: Conquering Suffering, Enriching Humanity, World Health Organization, Geneva, 1997.

2. World Health Organization: The World Health Report 1996: Fighting Disease, Fostering Development, World Health Organization, Geneva, 1996.

3. Woolf AD: The bone and joint decade 2000-2010. Ann Rheum Dis 59: 81, 2000.

4. Silverman SL: Quality of life issues in osteoporosis. Curr Rheum Rep 7: 39, 2005.

5. LiPS P, van SCHOOR NM: Quality of life with osteoporosis. Osteoporosis Int 16: 447, 2005.

6. Dolan P, Torgerson DJ: The cost of treating osteoporotic fractures in the UK female population. Osteoporosis Int 8: 611, 1998.

7. Department of Health: National Service Framework (NSF) for Older People, HMSO, London, 2001.

8. Dempster DW, Lindsay R: Pathogenesis of osteoporosis. Lancet 341: 797, 1993.

9. Mundy G: Maintaining the balance. ORGYN 12: 32, 2001.

10. PRINCE RL: How to diagnose the presence of osteoporosis and assess the risk of fracture. Best Pract Res Clin Rheumatol 15: 345, 2001.

11. Hakim A, Clunie G: Oxford Handbook of Rheumatology, p 254, Oxford University Press, Oxford, 2002.

12. Grey R: Avoiding the medico legal pit falls. Hosp Update 6: 53, 1998.

13. ANDERSON F: Best practice for oral steroid prescribing. Hosp Update 6 (suppl 2): S12, 1998.

14. Roben P, Barkmanns R, Ulrich S, ET al: Assessment of phalangeal bone loss in patients with rheumatoid arthritis by quantitative ultrasound. Ann Rheum Dis 60: 670, 2001.

15. Green MJ, Deodhar AA: Bone change in early rheumatoid arthritis. Best Pract Res Clin Rheumatol 15: 105, 2001.

16. Mikuls TR: Co-morbidity in rheumatoid arthritis. Best Pract Res Clin Rheumatol 17: 729, 2003.
17. HuUsko TM, Korpela M, Karppi P, ET AL: Threefold increased risk of hip fractures with rheumatoid arthritis in central Finland. Ann Rheum Dis 60: 521, 2001.

18. Nolla JM, Gomez-Vaquero C, Romera M, et al: Osteoporotic vertebral fracture in clinical practice: 669 patients diagnosed over a 10 year period. J Rheumatol 28: 2289, 2001.

19. Maenpaa HM, Soini I, Lehto MU, et al: Insufficiency fractures in patients with chronic inflammatory joint disease. Clin Exp Rheumatol 20: 77, 2002.

20. PERIS P: Stress fractures in rheumatological practice: clinical significance and localizations. Rheumatol Int $\mathbf{2 2}$ : 77, 2002.

21. Lems WF, Dijkmans BAC: Should we look for osteoporosis in patients with rheumatoid arthritis? Ann Rheum Dis 57: 325, 1998.

22. Brand CA, Jolley D, Tellus M, ET Al: Risk factors for osteoporosis and fracture in patients attending rheumatology outpatient clinics. Aust N Z J Med 29: 197, 1999.

23. Beardsworth A, Purdie D: "Screening and Bone Density," in Update: Menopause, 3rd Ed, p 33, Excerpta Medica, Oxford, 1999.

24. BRown P: A five step management plan for general practice. Osteoporosis Rev 9: 8, 2001.

25. LindSAY R: Pathogenesis of osteoporosis. Lancet 341: 801, 1993.

26. Аввот NC: Pointing the bone at osteoporosis. ORGYN 12: $22,2001$.

27. Nueh CF, Boivin CM, Gough A, ET AL: Evaluation of finger ultrasound in the assessment of bone status with application of rheumatoid arthritis. Osteoporosis Int 9: 82, 1999

28. ARnett FC, Edworth SM, Bloch DA, et AL: The American Rheumatism Association 1987 revised criteria for the classification of rheumatoid arthritis. Arthritis Rheum 31: 315, 1988.

29. White CE, Cooper RG: Prescribing and monitoring of disease-modifying anti-rheumatic drugs (DMARDS) for inflammatory arthritis. Rheum Dis Pract [serial online], 2002. Available at: http://www.arc.org.uk/about_arth/ med_reports/series4/ip/6508/6508.htm. Accessed February 5, 2007.

30. NJEH CF, GENANT HK: Bone loss: quantitative imaging techniques for assessing bone mass in rheumatoid arthritis. Arthritis Res 2: 446, 2000.

31. Ventura V, Mauloni M, Moura M, et al: Ultrasound velocity changes at the proximal phalanxes of the hand in pre-, peri- and postmenopausal women. Osteoporosis Int 6: 368, 1996.

32. Howard GM, Nguyen TV, Pocock NA, ET AL: Influence of handedness on calcaneal ultrasound: implications of the assessment of osteoporosis and study design. Osteoporosis Int 7: 190, 1997.

33. RANG HP, DAle MM, RitTer JM: Pharmacology, 4th Ed, p 416, Churchill Livingstone, London, 1999.

34. LUKERT BP, RAISZ LG: Glucocorticoid-induced osteoporosis: pathogenesis and measurement. Ann Intern Med 112: 352, 1990.

35. Churchill Medical Web site. Available at: http://www. Churchillmedical.co.uk. Accessed January 10, 2002.

36. Mazees RB: "Bone Densitometry," in Textbook of Nuclear Medicine, ed by MA Wilson, p 279, Raven Press, Philadelphia, 1998.

37. Pluijm SMF, Graafmans WC, Bouter LM, et al: Ultra-

Celebrating 100 years of continuous publication:1907-2007 
sound measurements for the prediction of osteoporotic fractures in the elderly. Osteoporosis Int 9: 550, 1999.

38. Imamoto K, HamanaKa Y, Yamamoto I, et AL: Correlation between the values of bone measurements using DXA, QCT and USD methods and the bone strength in calcanei in vitro. Acta Anat Nippon 73: 509, 1998.

39. Tromp AM, Smit JH, Deeg DJH, et AL: Quantitative ultrasound measurements of the tibia and the calcaneus in comparison with DXA measurements at various skeletal sites. Osteoporosis Int 9: 230, 1999.

40. Naganathan V, March L, Hunter D, et al: Quantitative heel ultrasound as a predictor for osteoporosis. Med J Aust 171: 297, 1999.

41. Hodgkinson R, NJeh CF, Currey JD, Et AL: The ability of ultrasound velocity to predict the stiffness of cancellous bone in vitro. Bone 21: 183, 1997.

42. BRook A, CoRbett M: Radiographic changes in early rheumatoid arthritis. Ann Rheum Dis 36: 72, 1977.

43. van der Heijde DM, van Leeuwen MA, van Riel PL, Et AL: Biannual radiographic assessments of hands and feet in a three year prospective follow-up of patients with early rheumatoid arthritis. Arthritis Rheum 35: 26, 1992.

44. Kobayashi S, Inoue S, Hosor T, et al: Association of BMD with polymorphism of the estrogen gene. J Bone Miner Res 11: 306, 1996.

45. Hulsmans HM, Jacobs JW, van der HeiJde DM: The course of radiologic damage during the first six years of rheumatoid arthritis. Arthritis Rheum 43: 1927, 2000.

46. Miller C, Nash DL: Foot problems and their conservative treatment in rheumatoid arthritis. Phys Med Rehabil Clin N Am 5: 747, 1994.

47. Michelson J, Easley M, Wigley FM, ET Al: Foot and ankle problems in rheumatoid arthritis. Foot Ankle Int 15: 608, 1994.

48. Klenerman L: The foot and ankle in rheumatoid arthritis. Br J Rheumatol 34: 443, 1995.

49. Cracchiolo A: Rheumatoid arthritis: hindfoot disease. Clin Orthop Relat Res 340: 58, 1997.

50. Gluer CC, Blake G, Lu Y, ET AL: Accurate assessment of precision errors: how to measure the reproducibility of bone densitometry techniques. Osteoporosis Int 5: 262, 1995.

51. Hausler KD, Rich PA, Smith PC, ET AL: Relationships between static histo-morphometry and ultrasound in the calcaneus. Calcified Tissue Int 64: 477, 1999.

52. Kerry RM, Holt GM, Stockley I: The foot in chronic rheumatoid arthritis: a continuing problem. The Foot 4: 201, 1994.

53. Mautalen CA, Oliveri B: "Densitometric Manifestations in Age-Related Bone Loss," in The Aging Skeleton, ed by CJ Rosen, J Glowacki, JP Bilezikian, p 263, Academic Press, London, 1999.

54. Fries J, Spitz P, Young D: Dimensions of health outcomes: the health assessment questionnaire, disability and pain scales. J Rheumatol 9: 789, 1982.

55. Budiman-Mak E, Conrad KJ, RoAch KE: The Foot Function Index: a measure of foot pain and disability. J Clin Epidemiol 44: 561, 1991.

56. Moots R, Jones N: Rheumatoid Arthritis: Your Questions Answered, p 48, Churchill Livingstone, Edinburgh, 2004.

57. SAMBrook PN: Corticosteroid osteoporosis. Z Rheumatol 59 (suppl 1): 45, 2000.

58. BAYLINK DJ: The diagnosis and management of osteoporosis. Z Rheumatol 59 (suppl 1): 42, 2000. 